\title{
SOME APPLICATIONS OF FRACTIONAL CALCULUS OPERATORS TO A NEW CLASS OF ANALYTIC FUNCTIONS WITH NEGATIVE COEFFICIENTS
}

\author{
E. KADIOĞLU
}

Atatürk University, Science and Art Faculty, Department of Mathematics, 25240 Erzurum, Turkey.

(Received Sep. 17, 1999; Revised Feb.2, 2000; Accepted Feb. 7, 2000)

\section{ABSTRACT}

The object of the present paper is to prove various distortion theorems for the fractional calculus of the functions in the class $P(n, \lambda, \alpha, r)$ consisting of analytic functions with negative coefficients in the unit disk.

\section{INTRODUCTION}

Let $A(n)$ denote the class of functions of the form

$$
f(z)=z-\sum_{k=n+1}^{\infty} \alpha_{k} z^{k}\left(\alpha_{k} \geq 0 ; n \in N=\{1,2, \ldots\}\right)
$$

which are analytic in the unit disk $U=\{z:|z|<1\}$. A function $f(z) \in A(n)$ is said to be in the class $P(n, \lambda, \alpha, r)$ if it satisfies

$$
\operatorname{Re}\left\{z \frac{\left(\lambda r z^{r-1}+1-\lambda\right) f^{\prime}(z)+\lambda z^{r} f^{\prime \prime}(z)}{\lambda z^{r} f^{\prime}(z)+(1-\lambda) f(z)}\right\}>\alpha,(r=1,2, \ldots)
$$

For some $\alpha(0 \leq \alpha<1), \lambda(0 \leq \lambda \leq 1)$ and for all $z \in U$. Some properties of the class $P(n, \lambda, \alpha, r)$ were investigated by Kamali and Kadioglu [3] .

Lemma 1. If a function $f(z) \in A(n)$ is in the class $P(n, \lambda, \alpha, r)$ then

$$
\sum_{k=n+1}^{\infty}[(k-\alpha)(\lambda k-\lambda+1)+\lambda k(r-1)] a_{k} \leq(1-\alpha)+\lambda(r-1)
$$

Proof. Let $\mathrm{f}(\mathrm{z}) \in \mathrm{P}(\mathrm{n}, \lambda, \alpha, r)$. We can write 


$$
\operatorname{Re}\left\{z \frac{\left(\lambda r z^{r-1}+1-\lambda\right) f^{\prime}(z)+\lambda z^{r} f^{\prime \prime}(z)}{\lambda z^{r} f^{\prime}(z)+(1-\lambda) f(z)}\right\}>\alpha
$$

Then

$$
\begin{gathered}
\frac{\left[\lambda r z^{r-1}+(1-\lambda)\right]\left[z-\sum_{k=n+1}^{\infty} k_{k} z^{k}\right]+\lambda z^{r+1}\left[-\sum_{k=n+1}^{\infty} k(k-1) a_{k} z^{k-2}\right]}{\lambda z^{r}\left[1-\sum_{k=n+1}^{\infty} k_{k} z^{k-1}\right]+(1-\lambda)\left[z-\sum_{k=n+1}^{\infty} a_{k} z^{k}\right]} \\
=\frac{\lambda r z^{r}+(1-\lambda) z-\sum_{k=n+1}^{\infty}\left[\lambda r k+\lambda k^{2}-\lambda k\right] a_{k} z^{k+r-1}-\sum_{k=n+1}^{\infty}(1-\lambda) k_{k} z^{k}}{\lambda z^{r}+(1-\lambda) z-\sum_{k=n+1}^{\infty}\left[\lambda k a_{k} z^{k+r-1}-\sum_{k=n+1}^{\infty}(1-\lambda) a_{k} z^{k}\right.}
\end{gathered}
$$

If we choose $z$ real and let $z \rightarrow 1^{-}$, we get

$$
\sum_{k=n+1}^{\infty}[(k-\alpha)(\lambda k-\lambda+1)+\lambda k(r-1)] a_{k} \leq(1-\alpha)+\lambda(r-1)
$$

\section{Fractional Calculus}

I begin with the statements of the following definitions of fractional calculus (that is, fractional derivatives and fractional integrals) which were defined by Owa $([4][5]$ and were used recently by Srivastava and Owa, Altintas, and Cho and Auof $([1],[2],[6])$.

Definition 2. The fractional integral of order $\delta$ is defined, for a function $f(z)$, by

$$
\mathrm{D}_{z}^{-\delta} f(z)=\frac{1}{\Gamma(\delta)} \int_{0}^{z} \frac{f(\zeta)}{(z-\zeta)^{1-\delta}} \mathrm{d} \zeta \quad(\delta>0)
$$

where $f(z)$ is an analytic function in a simply connected region of the $z$-plane containing the origin, and the multiplicity of $(z-\zeta)^{\delta-1}$ is removed by requiring $\log (z-\zeta)$ to be real when $z-\zeta>0$.

Definition 3. The fractional derivative of order $\delta$ is defined, for a function $\mathrm{f}(\mathrm{z})$, by

$$
\mathrm{D}_{\mathrm{z}}^{\delta} \mathrm{f}(\mathrm{z})=\frac{1}{\Gamma(1-\delta)} \frac{\mathrm{d}}{\mathrm{dz}} \int_{0}^{z} \frac{\mathrm{f}(\zeta)}{(\mathrm{z}-\zeta)^{\delta}} \mathrm{d} \zeta \quad(0 \leq \delta<1)
$$

where $f(z)$ is constrained, and the multiplicity of $(z-\zeta)^{-\delta}$ is removed, as in Definition 2.

Theorem 4. Let the function $f(z)$ be in the class $P(n, \lambda, \alpha, r)$. Then we have

$$
\left|D_{z}^{-\delta} f(z)\right| \geq \frac{|z|^{\delta}}{\Gamma(2+\delta)}\left\{|z|-\frac{[(1-\alpha)+\lambda(r-1)] \Gamma(n+2) \Gamma(2+\delta)}{[(n+1-\alpha)(\lambda n+1)+\lambda(n+1)(r-1)] \Gamma(n+2+\delta)}|z|^{n+1}\right\}
$$

and 


$$
\left|D_{z}^{-\delta} f(z)\right| \leq \frac{|z|^{\delta}}{\Gamma(2+\delta)}\left\{|z|+\frac{[(1-\alpha)+\lambda(r-1)] \Gamma(n+2) \Gamma(2+\delta)}{[(n+1-\alpha)(\lambda n+1)+\lambda(n+1)(r-1)] \Gamma(n+2+\delta)}|z|^{n+1}\right\}
$$

for $\delta>0$ and $z \in U$. The result is sharp.

Proof. It is easy to see that

$$
\Gamma(2+\delta) z^{-\delta} D_{z}^{-\delta} f(z)=z-\sum_{k=n+1}^{\infty} \frac{\Gamma(k+1) \Gamma(2+\delta)}{\Gamma(k+\delta+1)} a_{k} z^{k}=z-\sum_{k=n+1}^{\infty} \psi(k) a_{k} z^{k}
$$

where

$$
\psi(\mathrm{k})=\frac{\Gamma(\mathrm{k}+1) \Gamma(2+\delta)}{\Gamma(\mathrm{k}+\delta+1)}(\mathrm{k} \geq \mathrm{n}+1) .
$$

Noting that $\psi(k)$ is a decreasing function of $k$, we have

$$
0<\psi(\mathrm{k}) \leq \psi(\mathrm{n}+1)=\frac{\Gamma(\mathrm{n}+2) \Gamma(2+\delta)}{\Gamma(\mathrm{n}+2+\delta)} .
$$

Using Theorem 1, we have

$$
\begin{gathered}
{[(\mathrm{n}+1-\alpha)(\lambda \mathrm{n}+1)+\lambda(\mathrm{n}+1)(\mathrm{r}-1)] \sum_{\mathrm{k}=\mathrm{n}+1}^{\infty} a_{\mathrm{k}} \leq \sum_{\mathrm{k}=\mathrm{n}+1}^{\infty}[(\mathrm{k}-\alpha)(\lambda \mathrm{k}-\lambda+1)+\lambda \mathrm{k}(\mathrm{r}-1)] a_{\mathrm{k}}} \\
\leq(1-\alpha)+\lambda(\mathrm{r}-1),
\end{gathered}
$$

or

$$
\sum_{k=n+1}^{\infty} a_{k} \leq \frac{(1-\alpha)+\lambda(r-1)}{[(n+1-\alpha)(\lambda n+1)+\lambda(n+1)(r-)]}
$$

We can see that

$$
\begin{aligned}
\left|\Gamma(2+\delta) \mathrm{z}^{-\delta} \mathrm{D}_{\mathrm{z}}^{-\delta} \mathrm{f}(\mathrm{z})\right| & \geq|\mathrm{z}|-\psi(\mathrm{n}+1)|\mathrm{z}|^{\mathrm{n}+1} \sum_{\mathrm{k}=\mathrm{n}+1}^{\infty} a_{\mathrm{k}} \\
& \geq|\mathrm{z}|-\frac{[(1-\alpha)+\lambda(\mathrm{r}-1)] \Gamma(\mathrm{n}+2) \Gamma(2+\delta)}{[(\mathrm{n}+1-\alpha)(\lambda \mathrm{n}+1)+\lambda(\mathrm{n}+1)(\mathrm{r}-1)] \Gamma(\mathrm{n}+2+\delta)} \mid z^{\mathrm{n}+1}
\end{aligned}
$$

and

$$
\begin{aligned}
\left|\Gamma(2+\delta) z^{-\delta} D_{z}^{-\delta} f(z)\right| & \leq|z|+\psi(n+1)|z|^{n+1} \sum_{k=n+1}^{\infty} a_{k} \\
& \leq|z|+\frac{[(1-\alpha)+\lambda(r-1)] \Gamma(n+2) \Gamma(2+\delta)}{[(n+1-\alpha)(\lambda n+1)+\lambda(n+1)(r-1)] \Gamma(n+2+\delta)}|z|^{n+1}
\end{aligned}
$$

which prove the inequalities of the theorem. Further, equalities are attained for the function $\mathrm{f}(\mathrm{z})$ defined by

$$
D_{z}^{-\delta} f(z)=\frac{z^{\delta}}{\Gamma(2+\delta)}\left\{z-\frac{[(1-\alpha)+\lambda(r-1)] \Gamma(n+2) \Gamma(2+\delta)}{[(n+1-\alpha)(\lambda n+1)+\lambda(n+1)(r-1)] \Gamma(n+2+\delta)} z^{n+1}\right\}
$$

or

$$
f(z)=z-\frac{[(1-\alpha)+\lambda(r-1)]}{[(n+1-\alpha)(\lambda n+1)+\lambda(n+1)(r-1)]} z^{n+1} .
$$

Theorem 5. Let the function $f(z)$ be in the class $P(n, \lambda, \alpha, r)$. Then we have 


$$
\left|D_{z}^{\delta} f(z)\right| \geq \frac{|z|^{-\delta}}{\Gamma(2-\delta)}\left\{|z|-\frac{[(1-\alpha)+\lambda(r-1)] \Gamma(n+2) \Gamma(2-\delta)}{[(n+1-\alpha)(\lambda n+1)+\lambda(n+1)(r-1)] \Gamma(n+2-\delta)}|z|^{n+1}\right.
$$

and

$$
\left|D_{\cdot}^{\delta} f(z)\right| \leq \frac{|z|^{-\delta}}{\Gamma(2-\delta)}\left\{|z|+\frac{[(1-\alpha)+\lambda(r-1)] \Gamma(n+2) \Gamma(2-\delta)}{[(n+1-\alpha)(\lambda n+1)+\lambda(n+1)(r-1)] \Gamma(n+2-\delta)}|z|^{n+1}\right\}
$$

for $0 \leq \delta<1$ and $z \in U$. The result is sharp.

Proof. It is easy to see that

$$
\Gamma(2-\delta) \mathrm{z}^{\delta} \mathrm{D}_{\mathrm{z}}^{\delta} \mathrm{f}(\mathrm{z})=\mathrm{z}-\sum_{\mathrm{k}=\mathrm{n}+1}^{\infty} \frac{\Gamma(\mathrm{k}+1) \Gamma(2-\delta)}{\Gamma(\mathrm{k}-\delta+1)} a_{\mathrm{k}} \mathrm{z}^{\mathrm{k}}=\mathrm{z}-\sum_{\mathrm{k}=\mathrm{n}+1}^{\infty} \psi(\mathrm{k}) \mathrm{k} a_{\mathrm{k}} \mathrm{z}^{\mathrm{k}}
$$

where

$$
\psi(\mathrm{k})=\frac{\Gamma(\mathrm{k}) \Gamma(2-\delta)}{\Gamma(\mathrm{k}-\delta+\mathrm{l})} \quad(\mathrm{k} \geq \mathrm{n}+1)
$$

Noting that $\psi(k)$ is a decreasing function of $k$, we have

$$
0<\psi(\mathrm{k}) \leq \psi(\mathrm{n}+1)=\frac{\Gamma(\mathrm{n}+1) \Gamma(2-\delta)}{\Gamma(\mathrm{n}+2-\delta)} .
$$

Using Theorem 1, we have

$$
\frac{(\mathrm{n}+1-\alpha)(\lambda \mathrm{n}+1)+\lambda(\mathrm{n}+1)(\mathrm{r}-1)}{\mathrm{n}+1} \sum_{\mathrm{k}=\mathrm{n}+1}^{\infty} \mathrm{k} a_{\mathrm{k}} \leq(1-\alpha)+\lambda(\mathrm{r}-1)
$$

or

$$
\sum_{k=n+1}^{\infty} k a_{k} \leq \frac{(n+1)[(1-\alpha)+\lambda(r-1)]}{[(n+1-\alpha)(\lambda n+1)+\lambda(n+1)(r-1]} .
$$

We can see that

$$
\begin{aligned}
\left|\Gamma(2-\delta) z^{\delta} D_{z}^{\delta} f(z)\right| & \geq|z|-\psi(n+1)|z|^{n+1} \sum_{k=n+1}^{\infty} k a_{k} \\
& \geq|z|-\frac{[(1-\alpha)+\lambda(r-1)] \Gamma(n+2) \Gamma(2-\delta)}{[(n+1-\alpha)(\lambda n+1)+\lambda(n+1)(r-1)] \Gamma(n+2-\delta)}|z|^{n+1}
\end{aligned}
$$

and

$$
\begin{aligned}
\left|\Gamma(2-\delta) z^{\delta} D_{z}^{\delta} f(z)\right| & \leq|z|+\psi(n+1)|z|^{n+1} \sum_{k=n+1}^{\infty} k a_{k} \\
& \leq|z|+\frac{[(1-\alpha)+\lambda(r-1)] \Gamma(n+2) \Gamma(2-\delta)}{[(n+1-\alpha)(\lambda n+1)+\lambda(n+1)(r-1)] \Gamma(n+2-\delta)}|z|^{n+1}
\end{aligned}
$$

which prove the inequalities of the theorem. Further, equalities are attained for the function $\mathrm{f}(\mathrm{z})$ defined by

$$
D_{z}^{\delta} f(z)=\frac{z^{-\delta}}{\Gamma(2-\delta)}\left\{z-\frac{[(1-\alpha)+\lambda(r-1) \Gamma(n+2) \Gamma(2-\delta)}{[(n+1-\alpha)(\lambda n+1)+\lambda(n+1)(r-1)] \Gamma(n+2-\delta)} z^{n+1}\right.
$$

or 


$$
f(z)=z-\frac{[(1-\alpha)+\lambda(r-1)]}{[(n+1-\alpha)(\lambda n+1)+\lambda(n+1)(r-1)]} z^{n+1} .
$$

Remark: If we take $r=1$ in this paper, then we have the result given by Altıntaş [1]

\section{REFERENCES}

[1] Altıntas, O., On a subclass of certain starlike functions with negative coefficients, Math. Japon 36, No. 3, (1991), 489-495.

[2] Cho, N.E., M.K. Aouf., Some applications of fractional calculus operators to a certain subclass of analytic functions with negative coefficients, Tr. J. of Mat. 20 (1996), 553562.

[3] Kamali, M., E. Kadioglu., A new class of analytic functions with negative coefficients, Math. Balkanica (to be published).

[4] Owa, S., On the distortion theorems. I, Kyungpook Math. J. 18 (1978). 53-59.

[5] Owa, S., Some applications of fractional calculus, Research Notes in Math. 138, Pitman, Boston, London and Melbourne, (1985), 164-175.

[6] Srivastava, H.M., S. Owa., An applications of the fractional derivative, Math. Japon 29(1984), 383-389. 